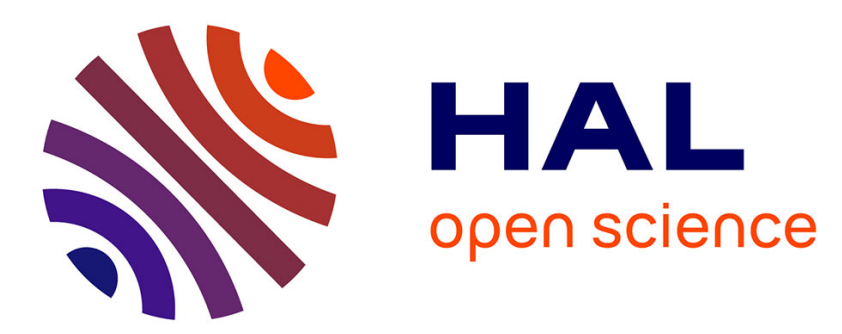

\title{
A mutual reference shape based on information theory
}

Stéphanie Jehan-Besson, C Tilmant, A de Cesare, A Lalande, A Cochet, Jean

Cousty, J Lebenberg, M Lefort, P Clarysse, Régis Clouard, et al.

\section{To cite this version:}

Stéphanie Jehan-Besson, C Tilmant, A de Cesare, A Lalande, A Cochet, et al.. A mutual reference shape based on information theory. International Conference on Image Processing ICIP, Oct 2014, Paris, France. pp.887 - 891, 10.1109/ICIP.2014.7025178 . hal-01081376

\section{HAL Id: hal-01081376 https://hal.science/hal-01081376}

Submitted on 7 Nov 2014

HAL is a multi-disciplinary open access archive for the deposit and dissemination of scientific research documents, whether they are published or not. The documents may come from teaching and research institutions in France or abroad, or from public or private research centers.
L'archive ouverte pluridisciplinaire HAL, est destinée au dépôt et à la diffusion de documents scientifiques de niveau recherche, publiés ou non, émanant des établissements d'enseignement et de recherche français ou étrangers, des laboratoires publics ou privés. 


\title{
A MUTUAL REFERENCE SHAPE BASED ON INFORMATION THEORY
}

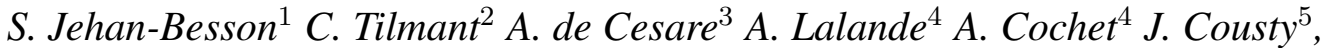

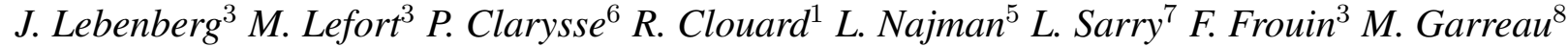 \\ ${ }^{1}$ CNRS, UMR 6072 GREYC, ENSICAEN, Université de Caen Basse Normandie, F-14050 Caen, France \\ 2 CNRS UMR 6602 ; Univ. Blaise Pascal, Institut Pascal, Clermont-Ferrand; ${ }^{3}$ Sorbonne Universités UPMC Paris 06, CNRS, INSERM, LIB, Paris, France \\ ${ }^{4}$ CNRS UMR 6306, Univ. Bourgogne, Le2I, Dijon; ${ }^{5}$ Univ. Paris Est, UMR 8049, Lab. Informatique Gaspard Monge, France \\ ${ }^{6}$ Univ. Lyon, CREATIS; CNRS UMR 5220; Inserm U1044 ; INSA-Lyon, Villeurbanne, France \\ ${ }^{7}$ CNRS UMR 6284 ; Univ. d'Auvergne, ISIT, Clermont-Ferrand; ${ }^{8}$ Inserm U1099 ; Univ. de Rennes 1, LTSI, Rennes, France
}

\begin{abstract}
In this paper, we propose to consider the estimation of a reference shape from a set of different segmentation results using both active contours and information theory. The reference shape is defined as the minimum of a criterion that benefits from both the mutual information and the joint entropy of the input segmentations and called a mutual shape. This energy criterion is here justified using similarities between information theory quantities and area measures, and presented in a continuous variational framework. This framework brings out some interesting evaluation measures such as the specificity and sensitivity. In order to solve this shape optimization problem, shape derivatives are computed for each term of the criterion and interpreted as an evolution equation of an active contour. Some synthetical examples allow us to cast the light on the difference between our mutual shape and an average shape. Our framework has been considered for the estimation of a mutual shape for the evaluation of cardiac segmentation methods in MRI.
\end{abstract}

Index Terms - Active contours, segmentation evaluation, shape gradient, average shape, cardiac MRI.

\section{INTRODUCTION}

Notions of shape averaging and shape statistics become particularly important when dealing with applications such as shape classification, registration, segmentation using shape prior or even segmentation evaluation. Indeed, in medical imaging, a ground truth is generaly not available to compare segmentation methods of anatomical structures. As far as the theoretical part is concerned, the shape averaging problem is closely related to shape optimization problems [1] and to the introduction of a shape metric. For example, such shape optimization algorithms have already been proposed in order to compute average shapes $[2,3]$ or more recently median shapes [4] by minimizing different shape metrics such as the Hausdorff distance [2] or the symmetric area difference be- tween shapes [3]. Another different technique for combining multiple segmentations or shapes is the STAPLE (Simultaneous Truth and Performance Level Estimation) algorithm proposed by Warfield et al. [5, 6]. Their algorithm consists in one instance of the Expectation Maximisation (EM) algorithm where the true segmentation is estimated by maximizing the likelihood of the complete data. Their pixel-wise approach leads to the estimation of a reference shape simultaneously with the sensitivity and specificity of each input segmentation. In this paper, we propose to introduce such a statistical modeling within a variational framework. Moreover, we take advantage of the analogies between information theory and area measures in order to estimate what we call a "mutual shape". We propose to maximize the mutual information between the $n$ input segmentations while minimizing their joint entropy. Such a statistical criterion can be interpreted as a robust measure of the symmetric area difference. The statistical model brings out both the sensitivity and specificity parameters and these parameters are estimated jointly with the reference mutual shape as in the STAPLE algorithm. However, the energy criterion is different from STAPLE and is justified using information theory quantities. Moreover, the advantage of our formalism is to exhibit both the domain and the associated contour. Compared with the STAPLE algorithm, such a continuous formalism may also be interesting in order to add some geometrical or photometric priors directly in the criterion to minimize. The mathematical framework is presented in section 2 . In section 3, the proposed algorithm is tested on both synthetic and medical cardiac MR images.

\section{MUTUAL SHAPE DEFINITION}

\subsection{Problem statement and statistical models}

Let us denote $\Omega$ the image domain and consider $\left\{\Omega_{1}, \Omega_{2}, \ldots, \Omega_{n}\right\}$ a family of $n$ shapes corresponding to $n$ different segmentations of the same unknown shape denoted by $\mu$. Each shape can be represented through a random binary label $D_{i}(X)$ whose observations are such that $d_{i}(x)=1$ if $x \in \Omega_{i}$ and 
$d_{i}(x)=0$ if $x \notin \Omega_{i}$. From all these segmented shapes, our goal is to compute a reference shape in order to mutualize the information of the $n$ segmented shapes. Let us denote by $T(X)$ the binary random variable associated with the unknown reference shape $\mu$ (with $\bar{\mu}$ the complementary region of $\mu$ ) such that $T(x)=1$ if $x \in \mu$ and $T(x)=0$ if $x \in \bar{\mu}$. The notation $t$ represents a realization of $T(X)$ and $|\Omega|$ the size of the image domain $\Omega$.

In order to estimate a reference shape, we exploit the analogies between information measures (mutual information, joint entropy) and area measures. In [7, 8], it is shown that the joint entropy $H(.,$.$) and the mutual information$ $I(.,$.$) can be interpreted in terms of area measures as follows:$

$H\left(D_{i}, T\right)=\operatorname{mes}\left(\tilde{D}_{i} \cup \tilde{T}\right), I\left(D_{i}, T\right)=\operatorname{mes}\left(\tilde{D}_{i} \cap \tilde{T}\right)$,

with $\tilde{X}$ the abstract set associated with the random variable $X$ and mes a signed measure defined on an algebra of sets. Let us first remind that an average shape [3] can be estimated using active contours by minimizing the sum of the union of the shapes $\Omega_{i}$ while maximizing the sum of the intersection according to $\mu$. By analogy with this criterion, the sum of the joint entropies (union of sets) will be minimized while the sum of the mutual information quantities (intersection of sets) will be maximized. In order to minimize a single criterion, we use the classic relation between mutual information and conditional entropy: $I\left(D_{i}, T\right)=H\left(D_{i}\right)-H\left(D_{i} / T\right)$. Since $H\left(D_{i} / T\right) \geq 0$ and $H\left(D_{i}\right)$ is independent of $T$, we will rather minimize $H\left(D_{i} / T\right)$. Due to all these considerations and properties, we propose to minimize the following criterion according to $T$ :

$$
E(T)=\sum_{i=1}^{n}\left(H\left(D_{i}, T\right)+H\left(D_{i} / T\right)\right) .
$$

In the sequel, the sum of joint entropies is denoted by $J H(T)=\sum_{i=1}^{n} H\left(D_{i}, T\right)$ and the sum of conditional entropies (coming from the mutual information) by $M I(T)=$ $\sum_{i=1}^{n} H\left(D_{i} / T\right)$. Note that this criterion is implicitly based on the assumption that random variables $D_{i}$ are considered as being independent.

\subsection{Continuous framework}

In order to take advantage of the previous statistical criterion (2) within a continuous shape optimization framework, we propose to express the joint and conditional probability density functions according to the reference shape $\mu$. Let us first express the conditional entropy :

$H\left(D_{i} / T\right)=-\sum_{t \in\{0,1\}} p(t) \sum_{d_{i} \in\{0,1\}} p\left(d_{i} / t\right) \log \left(p\left(d_{i} / t\right)\right)$,

with $p(T=t)=p(t)$ and $p\left(D_{i}=d_{i} / T=t\right)=p\left(d_{i} / t\right)$.

The conditional probability $p\left(d_{i}=1 / t=1\right)$ corresponds to the sensitivity parameter $p_{i}$ (true positive fraction):

$$
p_{i}(\mu)=p\left(d_{i}=1 / t=1\right)=\frac{1}{|\mu|} \int_{\mu} d_{i}(\mathbf{x}) d \mathbf{x} .
$$

The conditional probability $p\left(d_{i}=0 / t=0\right)$ corresponds to the specificity parameter $q_{i}$ (true negative fraction):

$$
q_{i}(\mu)=p\left(d_{i}=0 / t=0\right)=\frac{1}{|\bar{\mu}|} \int_{\bar{\mu}}\left(1-d_{i}(\mathbf{x})\right) d \mathbf{x} .
$$

The random variable $T$ takes the value 1 with a probability $p(t=1)=|\mu| /|\Omega|$ and 0 with a probability $p(t=0)=$ $|\bar{\mu}| /|\Omega|$. The $M I$ criterion can be expressed as follows:

$$
\begin{array}{r}
M I(\mu)=-\sum_{i=1}^{n} \frac{|\mu|}{|\Omega|}\left(\left(1-p_{i}\right) \log \left(1-p_{i}\right)+p_{i} \log p_{i}\right) \\
+\frac{|\bar{\mu}|}{|\Omega|}\left(q_{i} \log q_{i}+\left(1-q_{i}\right) \log \left(1-q_{i}\right)\right) .
\end{array}
$$

The parameters $p_{i}$ and $q_{i}$ depend explicitly on $\mu$, which must be taken into account in the optimization process.

Let us now express, according to $\mu$ and in a continuous setting $J H(T)$. The following expression of the joint entropy is considered:

$$
H\left(D_{i}, T\right)=-\sum_{t \in\{0,1\}} \sum_{d_{i} \in\{0,1\}} p\left(d_{i}, t\right) \log \left(p\left(d_{i}, t\right)\right),
$$

with $p\left(D_{i}=d_{i}, T=t\right)=p\left(d_{i}, t\right)$.

The following estimates for the joint probabilities are then used $(a=0$ or $a=1)$ :

$$
\begin{aligned}
& p\left(d_{i}=a, t=1\right)=\frac{1}{|\Omega|} \int_{\mu}\left((1-a)\left(1-d_{i}(\mathbf{x})\right)+a d_{i}(\mathbf{x})\right) d \mathbf{x}, \\
& p\left(d_{i}=a, t=0\right)=\frac{1}{|\Omega|} \int_{\bar{\mu}}\left((1-a)\left(1-d_{i}(\mathbf{x})\right)+a d_{i}(\mathbf{x})\right) d \mathbf{x} .
\end{aligned}
$$

The criterion $J H(\mu)$ can then be written as follows:

$$
\begin{aligned}
J H(\mu) & =-\frac{1}{|\Omega|} \sum_{i=1}^{n}\left[\int_{\mu}\left(\left(1-d_{i}\right) \log \left(A_{i}\right)+d_{i} \log \left(B_{i}\right)\right) d \mathbf{x}\right. \\
& \left.+\int_{\bar{\mu}}\left(\left(1-d_{i}\right) \log \left(A_{i}(\bar{\mu})\right)+d_{i} \log \left(B_{i}(\bar{\mu})\right)\right) d \mathbf{x}\right]+C,
\end{aligned}
$$

with $A_{i}(\mu)=\int_{\mu}\left(1-d_{i}(\mathbf{x})\right) d \mathbf{x}, A_{i}(\bar{\mu})=\int_{\bar{\mu}}\left(1-d_{i}(\mathbf{x})\right) d \mathbf{x}$, $B_{i}(\mu)=\int_{\mu} d_{i}(\mathbf{x}) d \mathbf{x}$ and $B_{i}(\bar{\mu})=\int_{\bar{\mu}} d_{i}(\mathbf{x}) d \mathbf{x}$. The term $C$ is equal to $n \log (\Omega)$ and is independent from $\mu$.

The global criterion (2) is then expressed according to $\mu$ using the expression of $J H(\mu)$ given in (10) and the expression of $M I(\mu)$ given in (6). We also add a regularization term denoted by $R(\partial \mu)$ in order to smooth the boundary:

$$
E(\mu)=J H(\mu)+M I(\mu)+\lambda R(\partial \mu),
$$

with $\lambda$ a positive parameter and $R(\partial \mu)=\int_{\partial \mu} d s$.

In this form, the minimization of such a criterion can be considered using active contours and shape gradients. 


\subsection{Optimization using shape gradients}

In order to compute a local minimum of the criterion (11), we propose to take advantage of the framework developed in [9] which is based on the shape optimization tools proposed in [1, Chap.8]. The main idea is to deform an initial curve (or surface) towards the boundaries of the object. After derivation, we find the following evolution equation:

$$
\left(\frac{\partial \Gamma}{\partial \tau}\right)=\left(v_{J H}+v_{M I}+\lambda \kappa\right) \mathbf{N}
$$

where $\kappa$ is the curvature of the contour balanced with the regularisation parameter $\lambda$. The velocities $v_{M I}$ and $v_{J H}$ are given by the following equations:

$v_{M I}=\frac{1}{|\Omega|} \sum_{i=1}^{n}\left[\left(1-d_{i}(\mathbf{x})\right) \log \left(\frac{q_{i}}{1-p_{i}}\right)+d_{i}(\mathbf{x}) \log \left(\frac{1-q_{i}}{p_{i}}\right)\right]$

$v_{J H}=\frac{1}{|\Omega|} \sum_{i=1}^{n}\left[d_{i}(\mathbf{x}) \log \left(\frac{B_{i}(\bar{\mu})}{B_{i}(\mu)}\right)+\left(1-d_{i}(\mathbf{x})\right) \log \left(\frac{A_{i}(\bar{\mu})}{A_{i}(\mu)}\right)\right]$

where $A_{i}$ and $B_{i}$ have been introduced in equation (10).

\section{MUTUAL SHAPE ESTIMATION}

This evolution equation has been tested on both synthetic and medical images.

\subsection{Synthetic images}

We propose a test sequence consisting of different segmentations of a lozenge (Fig.1). The first entry $\left(m_{1}\right)$ is the true segmentation mask, the other entries $\left(m_{2}, m_{3}, m_{4}, m_{5}\right)$ represent the segmentation of $1 / 4$ of the true lozenge (Fig.1(b)).

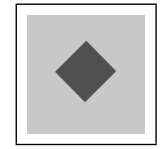

(a) Lozenge

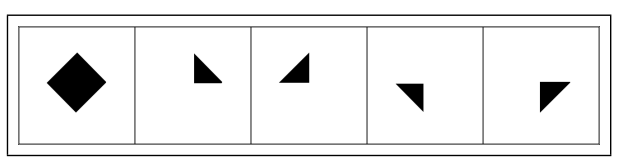

(b) $m_{1}, m_{2}, m_{3}, m_{4}, m_{5}$
Fig. 1. The test image (a) and (b) the different segmentation entries.

When computing the average of the different characteristic functions, we remark (Fig.2(b)) that some masks share an intersection. Indeed the values of the average image belong to the interval $[0,0.6]$. This average image $I_{A}$ is then binarized in (Fig.2(b)) with the threshold 0.5. This gives us a simple majority voting procedure.

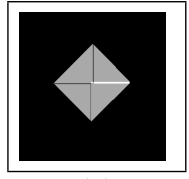

(a)

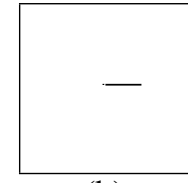

(b)
Fig. 2. The average image $I_{A}$ (a) and the corresponding binarized average image $I_{A T}$ (b) of the masks in Fig.1(b).
We then use the evolution equations of the mutual shape (12). The initial contour is chosen as a circle including the lozenge (Fig.3(a)). The mutual shape algorithm is able to recover the whole lozenge as shown in Fig.3(d). When using the mutual shape, the result is then different from the average shape given in Fig.2(b) which corresponds to a line.

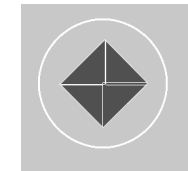

(a) It. 0

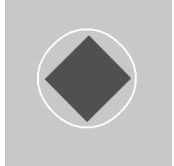

(b) It. 80

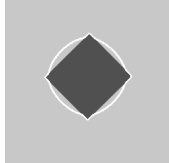

(c) It. 140

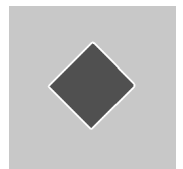

(d) Mutual shape
Fig. 3. Evolution using the mutual shape $(\lambda=10)$. In (a), the initial contour is in white (circle) and the other white lines represent the different segmentation entries. (Iterations are denoted by It.)

An outlier ( $m_{6}$ given Fig.4(a)) was introduced in the initial sequence of masks in order to test the robustness of the mutual shape estimation. Indeed, our goal is to test if the mutual shape is different from a simple union of the different masks. In Fig.4, the different steps of the evolution of the contour are displayed. The final contour (Fig.4(d)) fits the lozenge and excludes the outlier from the final contour.

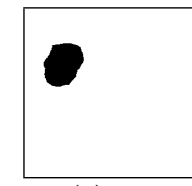

(a) $m_{6}$

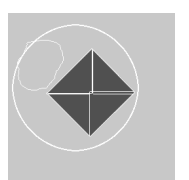

(b) It. 0

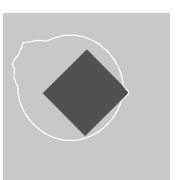

(c) It. 100

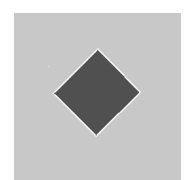

(d) Mutual shape
Fig. 4. Introduction of an outlier $\left(m_{6}\right)$ (a) in the initial sequence of masks (Fig.1(b)) and estimation of the mutual shape with $\lambda=10$. Iteration 100 is provided in (c).

When the active contour evolves using the evolution equation (12), the parameters $p_{i}$ and $q_{i}$ are estimated jointly with the mutual shape as proposed in STAPLE [5]. The evolution of these parameters is given in Table 1 for the initial contour, iteration 100 and the final contour. According to the final values reported in Table 1, we can conclude that the best segmentation corresponds to the shape $m_{1}$ with $p_{1}=1$ and $q_{1}=1$ and that the shape $m_{6}$ is an outlier since $p_{6}=0$. The other segmentations correspond to one quarter of the lozenge which leads to a sensitivity parameter around 0.25 .

\begin{tabular}{|c|c|c|c|c|c|c|c|}
\hline It. & & $m_{1}$ & $m_{2}$ & $m_{3}$ & $m_{4}$ & $m_{5}$ & $m_{6}$ \\
\hline It. 0 & $p_{i}$ & 0.35 & 0.09 & 0.09 & 0.08 & 0.09 & 0.15 \\
(Fig. 4.b) & $q_{i}$ & 1 & 1 & 1 & 1 & 1 & 1 \\
\hline It. 100 & $p_{i}$ & 0.60 & 0.15 & 0.15 & 0.13 & 0.16 & 0.27 \\
(Fig. 4.c) & $q_{i}$ & 1 & 1 & 1 & 1 & 1 & 1 \\
\hline Final & $p_{i}$ & 1 & 0.24 & 0.26 & 0.22 & 0.27 & 0 \\
(Fig. 4.d) & $q_{i}$ & 1 & 1 & 1 & 1 & 1 & 0.93 \\
\hline
\end{tabular}

Table 1. Joint evolution of the contour and of the values $p_{i}$ and $q_{i}$ for masks 1 to 6 . 


\subsection{Cardiac MR images}

The estimation of such a mutual shape is tested for the unsupervised evaluation of segmentation methods of the left ventricular cavity from cardiac cine-MRI. It takes place in a larger project on cardiac images segmentation evaluation developed in $[10,11,12]^{1}$. Two examples on two different MRI slices of the 2009 MICCAI challenge database (from the sequences SC-HYP-08 and SC-HYP-37) [13]. The slices are chosen due to the presence of outlier segmentations. The different segmentation entries are selected inside a database that contains the results obtained by different research teams $[14,15,16,17,18,19]$ for some parameters which are not necesseraly the optimal ones. The corresponding contours of the different entries are given respectively in Fig. 5 for the first slice (denoted by slice 1) and in Fig.7 for the second slice (denoted by slice 2). For the slice 2, the endocardium is not well delimited due to the presence of the aortic root which leads to two outlier segmentations.

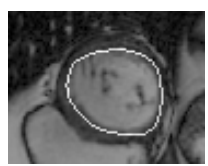

$m_{1}$

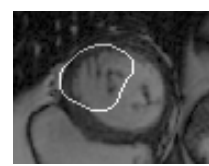

$m_{2}$

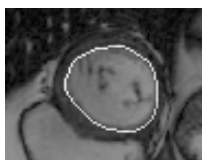

$m_{3}$

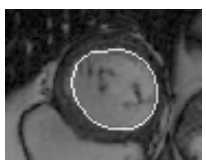

$m_{4}$

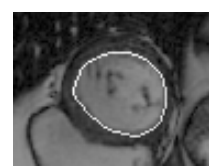

$m_{5}$
Fig. 5. The different segmentation methods $m_{1}$ to $m_{5}$ (slice 1).

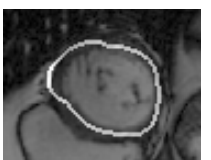

(a) Initial contour

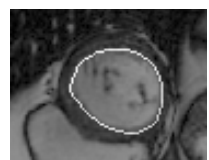

(b) Mutual shape

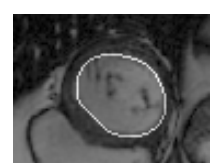

(c) Expert contour
Fig. 6. Estimation of the mutual shape (b) using the masks $m_{1}$ to $m_{5}$ (Fig.5) from the initial contour (a) (slice 1).

\begin{tabular}{|c|c|c|c|c|c|}
\hline i & $m_{1}$ & $m_{2}$ & $m_{3}$ & $m_{4}$ & $m_{5}$ \\
\hline pi & 1 & 0.440 & 0.998 & 0.913 & 0.982 \\
qi & 0.910 & 0.870 & 0.971 & 1 & 1 \\
\hline
\end{tabular}

Table 2. Sensitivity and specificity parameters $p_{i}$ and $q_{i}$ for the segmentations $m_{1}$ to $m_{5}$ displayed in Fig.5 (slice 1).

In Fig.6 and Fig.8, we give the corresponding estimated mutual shape in (b). The estimated mutual shape is computed using the 5 segmentation entries and the evolution equation (12) with the initial contour given in (a) and $\lambda=100$. The expert contour is also shown in (c) for each slice. For the slice 1,

\footnotetext{
${ }^{1}$ French project MediEval from GDR 2647 Stic-Santé (CNRS-INSERM)
}

the mutual contour is close to the expert one (Dice coefficient of 0.96). For the slice 2, the endocardium is not well delimited which leads to two outlier segmentations. In this case, we find a lower Dice coefficient of 0.84 . This example shows that our method is robust to outlier segmentations but not surprisingly the estimated mutual shape is dependent on the accuracy of the segmentation entries. During the curve evolution, the parameters $p_{i}$ and $q_{i}$ are estimated jointly with the mutual shape and allow to perform a classification of the performance level of each segmentation (see Tables 2 and 3). Since the best segmentation corresponds to $p_{i}=1$ and $q_{i}=1$, we can conclude that the obtained classification is visually coherent to its corresponding sequence of masks.

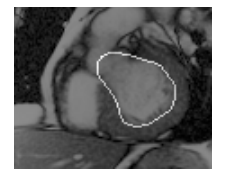

$m_{1}$

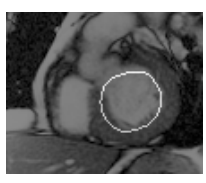

$m_{2}$

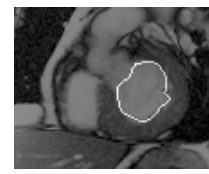

$m_{3}$

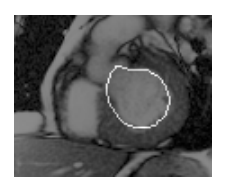

$m_{4}$

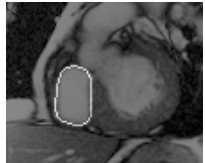

$m_{5}$
Fig. 7. The different segmentation methods $m_{1}$ to $m_{5}$ (slice 2).

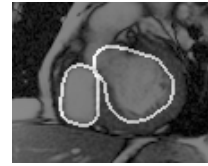

(a) Initial contour

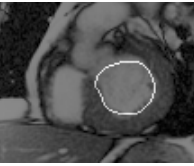

(b) Mutual shape

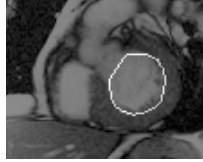

(c) Expert contour
Fig. 8. Estimation of the mutual shape (b) using the masks $m_{1}$ to $m_{5}$ (Fig.7) from the initial contour (a) (slice 2).

\begin{tabular}{|c|c|c|c|c|c|}
\hline & $m_{1}$ & $m_{2}$ & $m_{3}$ & $m_{4}$ & $m_{5}$ \\
\hline pi & 0.998 & 0.992 & 0.843 & 0.995 & 0 \\
qi & 0.844 & 0.956 & 0.983 & 0.945 & 0.801 \\
\hline
\end{tabular}

Table 3. Sensitivity and specificity parameters $p_{i}$ and $q_{i}$ for the segmentations $m_{1}$ to $m_{5}$ displayed in Fig.7 (slice 2).

\section{CONCLUSION}

In this paper, we propose to define and estimate what we call a mutual shape. Some experimental results are provided showing the applicability of this framework for segmentation evaluation without a reference shape. Our work in progress concerns the comparison of our method to STAPLE algorithm using the statistical validation proposed in [20] and extended validation tests on a larger database of cardiac cine-MR images. 


\section{REFERENCES}

[1] M.C. Delfour and J.P. Zolésio, Shapes and Geometries: Metrics, Analysis, Differential Calculus, and Optimization, Advances in design and control. SIAM, Philadelphia, PA, USA, 2001.

[2] G. Charpiat, O. Faugeras, and R. Keriven, "Approximations of shape metrics and application to shape warping and empirical shape statistics," FoCM, vol. 5, pp. 1-58, 2005.

[3] S. Soatto and A.J. Yezzi, "Deformotion: Deforming motion, shape average and the joint registration and segmentation of images," IJCV, vol. 53, pp. 153-167, 2002.

[4] B. Berkels, G. Linkmann, and M. Rumpf, "An SL(2) invariant shape median," JMIV, vol. 37 , no. 2, pp. 8597, 2010

[5] S.K. Warfield, K. H. Zou, and W. M. Wells III, "Simultaneous truth and performance level estimation (STAPLE): an algorithm for the validation of image segmentation," IEEE TMI, vol. 23, no. 7, pp. 903-921, 2004.

[6] O. Commowick, A. Akhondi-Asl, and S.K. Warfield, "Estimating a reference standard segmentation with spatially varying performance parameters," IEEE TMI, vol. 31 , no. 8, pp. 1593-1606, august 2012.

[7] F. M. Reza, An Introduction to Information Theory, pp. 106-108, McGraw-Hill, 1994.

[8] R. W. Yeung, "A new outlook on shannon's information measures," IEEE TIT, vol. 37, no. 3, pp. 466-474, 1991.

[9] G. Aubert, M. Barlaud, O. Faugeras, and S. JehanBesson, "Image segmentation using active contours : Calculus of variations or shape gradients," SIAM App. Math., vol. 63, pp. 2128-2154, 2003.

[10] S. Jehan-Besson, C. Tilmant, A. De Cesare, F. Frouin, L. Najman, A. Lalande, L. Sarry, C. Casta, P. Clarysse, C. Constantidinès, J. Cousty, M. Lefort, A. Cochet, and M. Garreau, "Estimation d'une forme mutuelle pour l'évaluation de la segmentation en imagerie cardiaque," GRETSI, 2011.

[11] A. Lalande, J. Lebenberg, I. Buvat, P. Clarysse, C. Casta, A. Cochet, C. Constantinidès, J. Cousty, A. de Cesare, S. Jehan-Besson, M. Lefort L. Najman, E. Roullot, L. Sarry, C. Tilmant, M. Garreau, and F. Frouin, "A reference free approach for the comparative evaluation of eight segmentation methods for the estimation of the left ventricular ejection fraction in cardiac MRI," in ESMRMB, 2012, p. 658.
[12] J. Lebenberg, I. Buvat, A. Lalande, P. Clarysse, C. Casta, A. Cochet, C. Constantinidès, J. Cousty, A. de Cesare, S. Jehan-Besson, M. Lefort, L. Najman, E. Roullot, L. Sarry, C. Tilmant, M. Garreau, and F. Frouin, "Non supervised ranking of different segmentation approaches: application to the estimation of the left ventricular ejection fraction from cardiac cine MRI sequences," IEEE TMI, vol. 31, no. 8, pp. 1651-1660, 2012.

[13] P. Radau, Y. Lu, K. Connelly, G. Paul, A.J. Dick, and G.A. Wright, "Evaluation framework for algorithm segmenting short axis cardiac MRI.," The MIDAS Journal, 2009.

[14] C. Constantinides, R. El Berbari, A. de Cesare, Y. Chenoune, E. Roullot, A. Herment, E. Mousseaux, and F. Frouin, "Development and evaluation of an algorithm for the automated segmentation of the left and right ventricles on cine MRI," IRBM, vol. 30, no. 4, pp. 188-191, 2009.

[15] J. Cousty, L. Najman, M. Couprie, S. ClémentGuinaudeau, T. Goissen, and J. Garot, "Segmentation of 4D cardiac MRI: automated method based on spatiotemporal watershed cuts," IVC, vol. 28, no. 8, pp. 12291243, 2010.

[16] J. Fleureau, M. Garreau, D. Boumier, and A. Hernandez, "3D multi-object segmentation of cardiac MSCT imaging by using a multi-agent approach," in IEEE EMBS, 2007, pp. 6004-7.

[17] J. Fleureau, M. Garreau, A. Simon, R. Hachemani, and D. Boulmier, "Assessment of global cardiac function in MSCT imaging using fuzzy connectedness segmentation," in Computers in Cardiology, 2008, pp. 725-728.

[18] A. Lalande, N. Salve, A. Comte, M.-C. Jaulent, L. Legrand, P.M. Walker, Y. Cottin, J.E. Wolf, and F. Brunotte, "Left ventricular ejection fraction calculation from automatically selected and processed diastolic and systolic frames in short axis cine-MRI," JCMR, vol. 6, no. 4, pp. 817-827, 2004.

[19] J. Schaerer, C. Casta, J. Pousin, and P. Clarysse, "A dynamic elastic model for segmentation and tracking of the heart in MR image sequences," MIA, vol. 14, no. 6, pp. 738-749, 2010.

[20] J. Lebenberg, F. Frouin, P. Clarysse, I. Buvat, C. Casta, A. Cochet, C. Constantinidès, J. Cousty, A. De Cesare, S. Jehan-Besson, M. Lefort, L. Najman, E. Roullot, L. Sarry, C. Tilmant, A. Lalande, and M. Garreau, "Combining multiple automated segmentations of the left ventricle in cardiac MRI: a statistical validation," in MAGMA, october 2013, vol. 26 supp. 1, p. 109. 